\title{
Activation of cell-surface proteases promotes necroptosis, inflammation and cell migration
}

\author{
Zhenyu Cai ${ }^{1}$, Anling Zhang ${ }^{1}$, Swati Choksi ${ }^{1}$, Weihua $\mathrm{Li}^{2}$, Tao $\mathrm{Li}^{2}$, Xue-Min Zhang ${ }^{2}$, Zheng-Gang Liu ${ }^{1}$ \\ ${ }^{I}$ Center for Cancer Research, National Cancer Institute, National Institutes of Health, Bethesda, MD 20892, USA, ${ }^{2}$ Institute of Basic \\ Medical Sciences, National Center of Biomedical Analysis, 27 Tai-Ping Road, Beijing 100850, China
}

\begin{abstract}
Necroptosis is a programmed, caspase-independent cell death that is morphologically similar to necrosis. TNF-induced necroptosis is mediated by receptor-interacting protein kinases, RIP1 and RIP3, and the mixed lineage kinase domain-like (MLKL). After being phosphorylated by RIP3, MLKL is translocated to the plasma membrane and mediates necroptosis. However, the execution of necroptosis and its role in inflammation and other cellular responses remain largely elusive. In this study, we report that MLKL-mediated activation of cell-surface proteases of the a disintegrin and metalloprotease (ADAM) family promotes necroptosis, inflammation and cell migration. ADAMs are specifically activated at the early stage of necroptosis when MLKL is phosphorylated and translocated to the cell plasma membrane. Activation of ADAMs induces ectodomain shedding of diverse cell-surface proteins including adhesion molecules, receptors, growth factors and cytokines. Importantly, the shedding of cell-surface proteins disrupts cell adhesion and accelerates necroptosis, while the soluble fragments of the cleaved proteins trigger the inflammatory responses. We also demonstrate that the shedding of E-cadherin ectodomain from necroptotic cells promotes cell migration. Thus, our study provides a novel mechanism of necroptosis-induced inflammation and new insights into the physiological and pathological functions of this unique form of cell death.
\end{abstract}

Keywords: MLKL; ADAM metalloprotease; necroptosis; inflammation

Cell Research (2016) 26:886-900. doi:10.1038/cr.2016.87; published online 22 July 2016

\section{Introduction}

Necroptosis is a programmed form of necrosis, characterized by the rounding of the cell, gaining in cell volume, rupture of plasma membrane and release of intracellular contents $[1,2]$. Distinct from apoptosis, which is executed by proteases of the caspase family, necroptosis is thought to be a form of protease-independent cell death. Recently, our understanding of the molecular mechanism of necroptosis has been greatly improved through the study of TNF-induced necroptosis. It is now known that receptor-interacting protein kinases RIPK1 (or RIP1) and RIPK3 (or RIP3), and the mixed lineage kinase domain-like (MLKL) constitute the core of the necroptosis machinery [3-9]. RIP1 recruits RIP3 to

Correspondence: Zheng-Gang Liu

Tel: +1-301-435-6351; Fax: +1-301-402-1997

E-mail: zgliu@helix.nih.gov

Received 12 May 2016; revised 20 June 2016; accepted 21 June 2016; published online 22 July 2016 initiates the formation of necrosome and activates RIP3 through phosphorylation upon necroptotic stimuli $[3,8$, 9]. Subsequently, activated RIP3 recruits and phosphorylates MLKL $[4,5]$. The phosphorylated MLKL in turn oligomerizes and translocates to the plasma membrane [10-13]. The plasma membrane translocation of MLKL is essential for increasing plasma membrane permeability. It has been suggested that MLKL mediates the disruption of plasma membrane permeability either by activating ion channels or forming pore structures directly in the plasma membrane [10-13]. In the former case, MLKL affects cell function and membrane permeability by association with specific ion channel target $[10$, 11]. In the latter case, MLKL binds to certain plasma membrane lipids and disrupts liposome integrity in vitro $[12,13]$. Although MLKL is thought to be an execution effector in the necroptosis pathway, the implementation of necroptosis and its role in inflammation and other cellular responses remain largely elusive. Particularly, the involvement of proteases in necroptosis has been neglected since it is believed that protease activation is not 
a part of the necroptotic process. In this study, we report that MLKL-mediated activation of cell-surface proteases of the a disintegrin and metalloprotease (ADAM) family promotes necroptosis, inflammation and cell migration.

\section{Results}

Necroptotic stimuli induce the cleavage of E-cadherin on cell surface

When cells undergo necroptosis, they round up, swell and then lose plasma membrane integrity $[1,14]$. We noticed that adherent cells, such as human intestinal epithelial HT29 cells, first lost cell-cell contact before they rounded up during necroptosis induced by TNF, Smac mimetic, and caspase inhibitor z-VAD-fmk (TSZ) treatment (Figure 1A). Since E-cadherin is a major transmembrane protein of the adherence junction between neighboring epithelial cells [15], we examined whether E-cadherin expression is altered during necroptosis. Surprisingly, we found that the full-length $120 \mathrm{kDa}$ E-cadherin protein was cleaved and yielded a $37-\mathrm{kDa}$ C-terminal fragment after $2 \mathrm{~h}$ TSZ treatment (Figure 1B), well before cell membrane integrity was disrupted (Figure 1A). Because the $80 \mathrm{kDa}$ ectodomain of E-cadherin was detected in the culture medium of TSZ-treated cells, E-cadherin was likely cleaved extracellularly at the cell surface (Figure 1C). The loss of E-cadherin from cell surface during necroptosis was further confirmed by immunofluorescence staining (Supplementary information, Figure S1A). In addition, E-cadherin cleavage was also induced by other types of necroptotic stimulus including Fas ligand or TRAIL combined with Smac mimetic and $z$-VAD-fmk or TNF- $\alpha$, cycloheximide, and z-VAD-fmk (TCZ; Supplementary information, Figure S1B-S1D). The cleavage of E-cadherin was specifically detected in necroptotic cells after TSZ treatment but not in apoptotic cells after TNF and Smac mimetic (TS) treatment (Figure 1D and Supplementary information, Figure S1E) or staurosporine treatment (Supplementary information, Figure S1F) or autophagic cells induced by rapamycin treatment (Supplementary information, Figure S1G). Both RIP1 and MLKL were required for E-cadherin cleavage, because treating HT29 cells with necrostatin-1, a RIP1 inhibitor [16] (Figure 1E) or knocking down MLKL in HT29 cells blocked the cleavage of E-cadherin (Figure 1F). Since RIP3 phosphorylates MLKL [4, 5], we then examined whether RIP3-mediated phosphorylation of MLKL induces the cleavage of E-cadherin. We found that co-expression of MLKL and RIP3 specifically induced the loss of E-cadherin expression in HEK293 cells, but did not affect the levels of GFP protein or the cell-surface calcium channel protein, ORAI-
1-YFP [17] (Figure 1G). Importantly, when MLKL was co-expressed with the kinase-dead RIP3 or when the two main RIP3-phosphorylation sites of MLKL were mutated (MLKL-T357A/S358A), the expression of E-cadherin was no longer changed (Figure $1 \mathrm{H}$ ). Collectively, these data suggest that necroptotic stimulus induces the cleavage of E-cadherin from cell surface through a MLKL-dependent mechanism.

Necroptotic stimuli induce a general ectodomain shedding of cell-surface proteins at the early stage of necroptosis

Since cadherins are a large family of cell-cell adhesion molecules [18], we then examined whether other proteins of this family are also cleaved in non-epithelial cells upon necroptotic induction. Using a pan-cadherin antibody recognizing the conserved $\mathrm{C}$-terminal region of all cadherins, we observed the cleavage of a cadherin protein, most likely $\mathrm{N}$-cadherin $[19,20]$, in human Jurkat $^{\mathrm{FADD}^{-/-}}$cells and mouse embryonic fibroblasts (MEFs; Figure 2A and 2B). Interestingly, we also found that the levels of the plasma membrane protein ATIA/ vasorin [21] were significantly decreased in MEFs and cadherin-negative L929 cells [22] following necroptotic stimulation (Figure $2 \mathrm{~B}$ and $2 \mathrm{C}$ ). These results suggest that the cleavage of plasma membrane proteins may be a general phenomenon at the early stage of necroptosis when cells are still intact. To explore this possibility, we further analyzed the extent of protein shedding from cell surface during necroptosis. We found that total protein levels were dramatically increased in the medium of TSZ-treated cells compared with that of non-treated cells at $4 \mathrm{~h}$ before cell plasma membrane became permeable (Figure 2D). Importantly, MLKL knockdown blocked the increase of the protein levels in the medium of treated cells (Figure 2D). Using mass spectrometry, we identified a variety of cell-surface proteins from the medium of HT29 cells treated with TSZ for $4 \mathrm{~h}$ (Figure 2E). The cleavage of some of these proteins including epithelial cell adhesion molecule (EpCAM), basal cell adhesion molecule (BCAM) and transferrin receptor (TfR) was confirmed by western blotting (Figure 2F). Furthermore, knockdown of MLKL prevented the cleavage of these proteins (Figure 2F). Thus, our data reveal a general ectodomain shedding of cell-surface proteins at the early stage of necroptosis.

Activation of ADAM metalloproteinases mediates shedding of cell-surface proteins in necroptosis

We then tested whether protease inhibitors could block the cleavage of E-cadherin in necroptosis. Addition of GM6001, a broad spectrum matrix metalloproteinase 
A
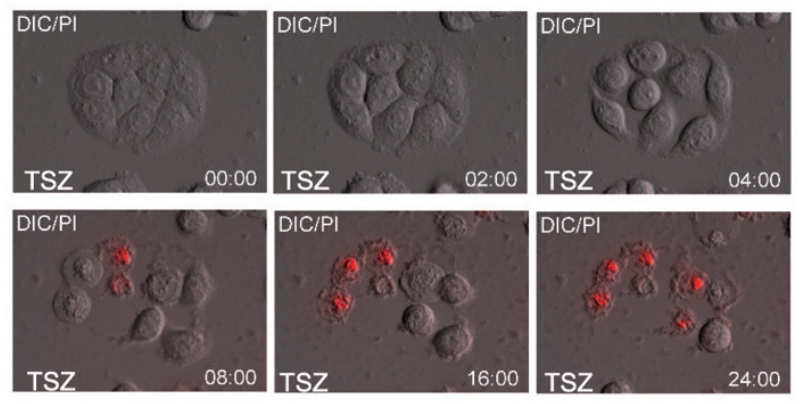

C

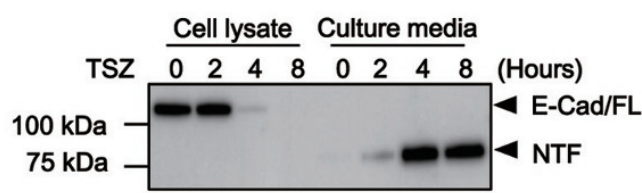

IB: Anti-E-Cad (N-terminal)

E

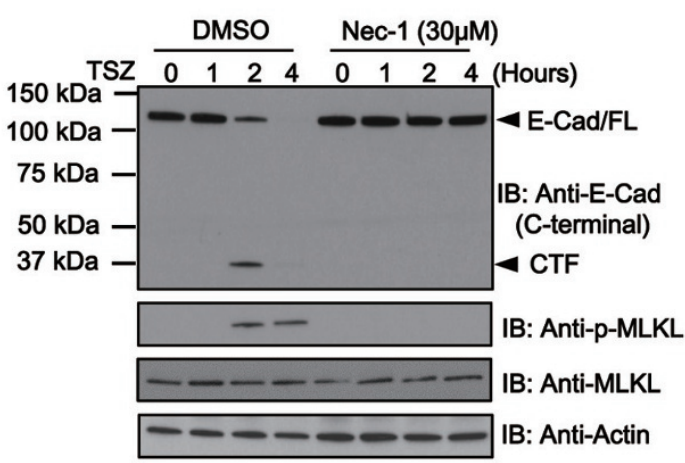

F
B

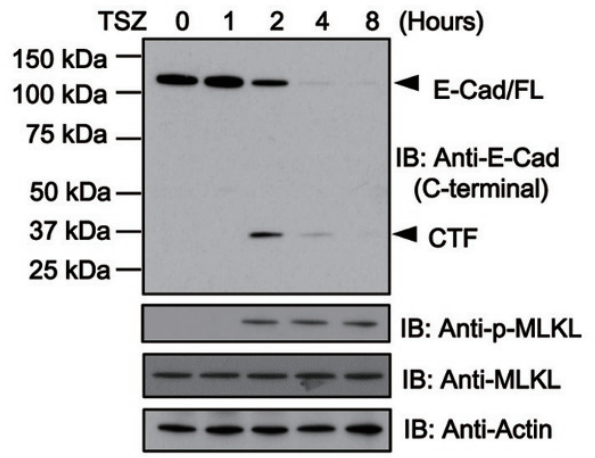

D
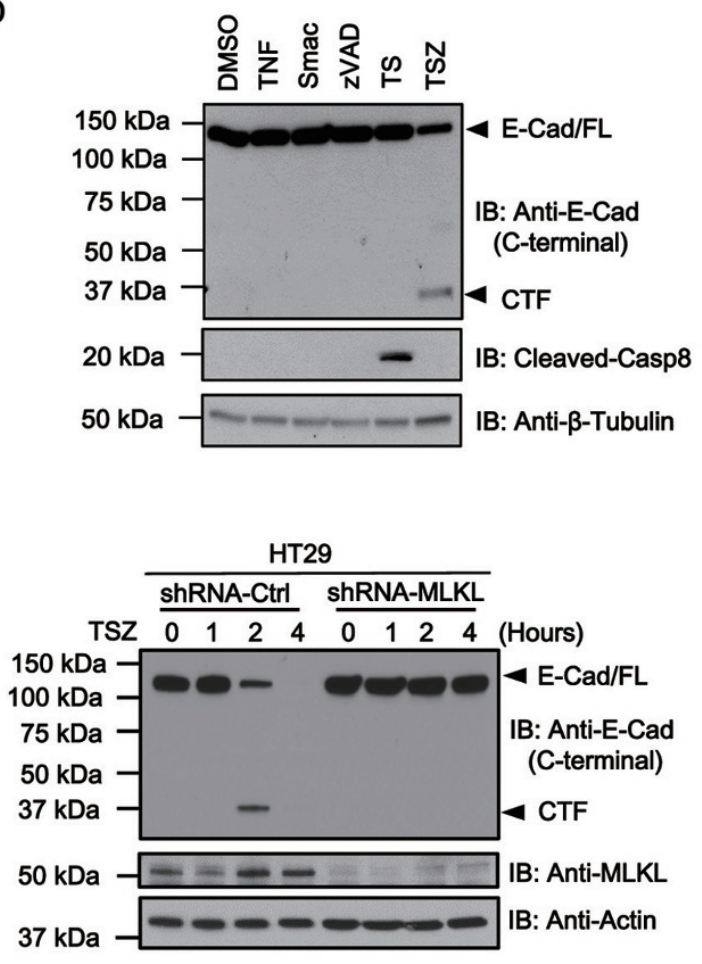

H

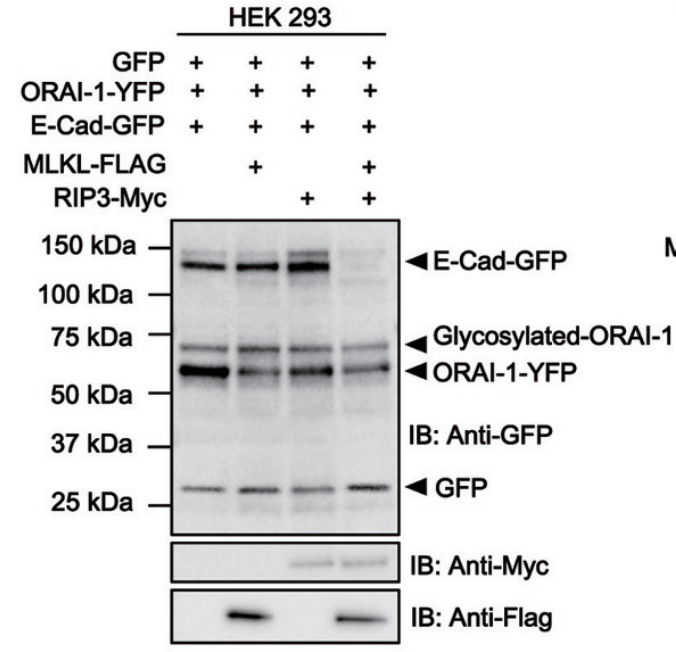


Figure 1 Necroptotic stimulus induces the cleavage of E-cadherin on cell surface. (A) Time-lapse microscopic analysis of cellular morphology (DIC, differential interference contrast) and PI uptake (red, indicating plasma membrane permeability) after induction of necroptosis by TSZ (TNF, Smac mimetic, Z-VAD-fmk) in HT29 cells. (B) HT29 cells were treated with TSZ at different time points as indicated. Cell lysates (B) or (C) conditioned medium was analyzed by immunoblotting with the indicated antibodies. (D) HT29 cells were treated with TNF alone, Smac mimetic alone, z-VAD-fmk alone, TNF and Smac mimetic (TS) or TNF, Smac mimetic and z-VAD-fmk (TSZ) for $4 \mathrm{~h}$. Cell lysates were analyzed by immunoblotting with the indicated antibodies. (E) HT29 cells were pre-treated with or without necrostatin-1 (Nec-1) and then treated with TSZ for 1, 2 and 4 h. Cell lysates were analyzed by immunoblotting as indicated. (F) shRNA-control or shRNA-MLKL HT29 cells were treated with TSZ for different time points. Cell lysates were analyzed by immunoblotting with the indicated antibodies. (G) HEK293 cells were transfected with GFP, ORAI-1-YFP, E-Cadherin-GFP, RIP3-V5 and MLKL-FLAG constructs as indicated. After 24 h, cell lysates were analyzed by immunoblotting with the indicated antibodies. (H) HEK293 cells were transfected with E-Cadherin-GFP, RIP3-V5, RIP-kinase dead-V5 (RIP3-KD-V5), MLKL-FLAG and MLKL phospho-mutated (MLKL-T357A/S358A) constructs as indicated. After $24 \mathrm{~h}$, cell lysates were analyzed by immunoblotting with the indicated antibodies. Data shown are representative of three independent experiments.

(MMP) inhibitor, prevented TSZ-induced cleavage of E-cadherin, while other protease inhibitors including a cysteine protease inhibitor (Z-LL)2 ketone, a serine protease inhibitor 3,4-DCI, and a protease inhibitor cocktail with a broad inhibition activities for multiple proteases did not prevent E-cadherin from cleavage (Figure 3A). Similarly, only MMP inhibitor GM6001 blocked TSZ-induced degradation of ATIA/vasorin in MEFs (Supplementary information, Figure 2A). Since E-cadherin of the non-treated cells is not cleaved when the cells were incubated with the medium of TSZ-treated cells (Supplementary information, Figure 2B), the cleavage of cell-surface proteins was most likely due to the activation of cell-surface proteases. Particularly, since it has been shown that ADAM10, a member of the plasma membrane-bound ADAM metalloproteinase family, cleaves E-cadherin [23], we then investigated whether ADAMs were responsible for the cleavage of cell-surface proteins. Indeed, both broad and specific ADAM inhibitors, including TAPI-2, GI254023 and GW280264X, all significantly inhibited the TSZ-induced cleavage of E-cadherin, EpCAM, TfR and BCAM in HT29 cells (Figure 3B). In MEFs or L929 cells, the degradation of ATIA and N-cadherin was also effectively blocked by the dual ADAM10 and ADAM17 inhibitor GW280264X (Supplementary information, Figure S2C and S2D). We then tested if the ADAMs are activated during necroptosis. Using a quenched fluorogenic peptide that is a specific substrate of ADAMs [24], we observed a significant increase of the metalloprotease activity after TSZ treatment in both HT29 cells and MEFs (Figure 3C and Supplementary information, Figure S2E), while such activation was not detected in MLKL knockdown or knockout cells (Figure 3D and Supplementary information, Figure S2F).

Among the 19 human ADAMs, 12 are known to be active metalloprotease [25]. We found that active metalloproteases ADAM9, ADAM10, ADAM15 and ADAM17 are expressed in HT29 cells (Supplementary information, Figure S3A) and knocked down each of them (Supplementary information, Figure S3B). While knockdown of ADAM15 or ADAM17 had no effect on the cleavage of cell-surface protein, knocking down ADAM9 or ADAM10 partially prevented the cleavage of E-cadherin and BCAM and had a minor protection on the cleavage of EpCAM (Figure 3E). ADAM10 knockdown also blocked TSZ-induced cleavage of TfR (Figure 3E). Importantly, knockdown of both ADAM9 and ADAM10 by two different sets of non-overlapping shRNAs inhibited the cleavage of cell-surface proteins more efficiently (Figure 3F and Supplementary information, Figure S3C). Therefore, ADAM9 and ADAM10 are critical for TSZ-induced cleavage of cell-surface proteins in HT29 cells. Similar results were also observed in human Jur$\mathrm{kat}^{\mathrm{FADD}^{-/}}$cells (Supplementary information, Figure S3D). In MEFs or L929 cells, we found that ADAM10 and ADAM17 were critical for the cleavage of cell-surface proteins since the double knockout of ADAM10/17 by CRISPR-Cas9 technology efficiently inhibited TSZ-induced cleavage (Figure 3G and Supplementary information, Figure S3E and S3F). Collectively, our data indicate that multiple ADAM metalloproteinases are activated to mediate the shedding of cell-surface proteins in response to necroptotic stimuli.

\section{MLKL forms complex with ADAMs to mediate activation of ADAM metalloproteases}

Since phosphorylated MLKL locates to the plasma membrane where functional ADAMs reside, we then examined whether MLKL is in the same complex with ADAMs following necroptosis induction. We first expressed MLKL, RIP3 and ADAM10 in HEK293 cells and examined the potential interaction between ADAM10 and MLKL or RIP3 by immunoprecipitation with N-terminal specific ADAM10 antibody. We found that MLKL was co-precipitated with ADAM10 only in the presence of RIP3, and however, no RIP3 was pulled down (Figure 
A

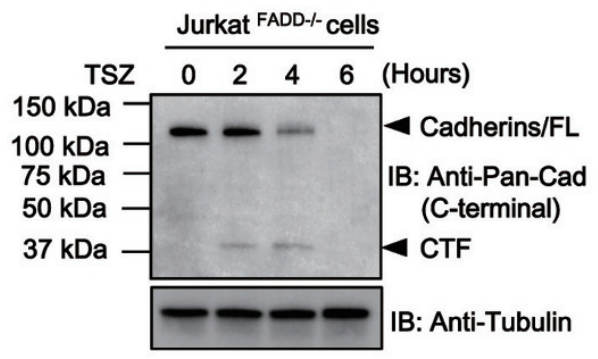

B

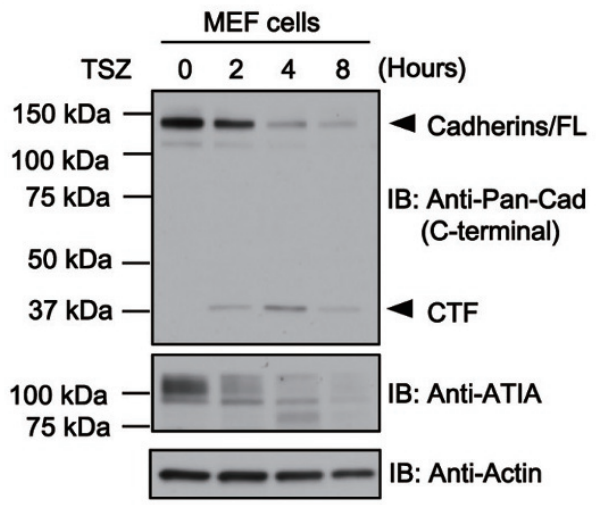

C

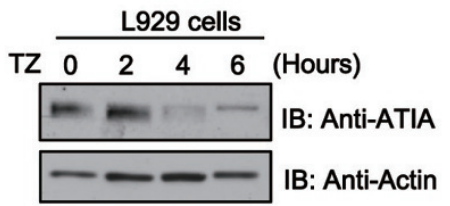

D

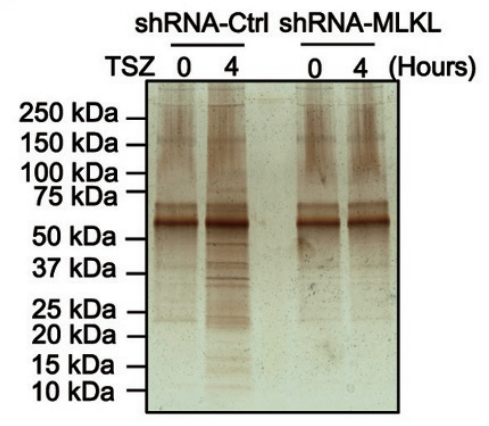

E

Cell adhesion molecules

E-Cadherin
Junctional adhesion molecule A
Basal cell adhesion molecule
Epithelial cell adhesion molecule
Desmoglein-2
Calsyntenin-1
Carcinoembryonic antigen-related cell adhesion
molecule 1
Junction plakoglobin
Podocalyxin
Syndecan-1
CD44

Cell surface receptors and ligands

Transferrin receptor Endothelial protein $\mathrm{C}$ receptor EPH receptor B4

Ephrin-B1

CD91

Ion channels

Chloride intracellular channel protein 1 Chloride intracellular channel protein 4

$\mathbf{F}$

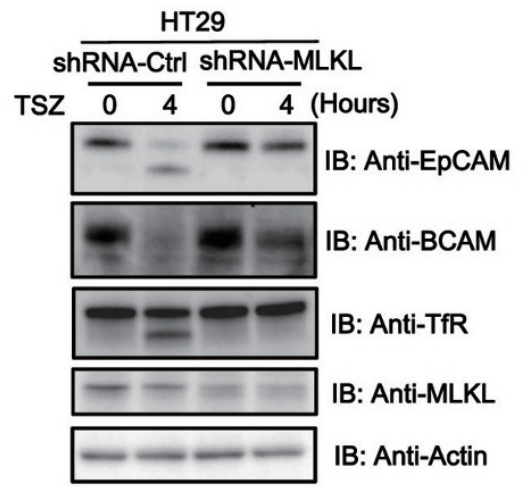

Figure 2 Necroptotic stimulus induces a general ectodomain shedding of cell-surface proteins. (A) Jurkat ${ }^{\mathrm{FAD}^{-/}}$cells or (B) MEFs were treated with TSZ for 2, 4 and $6 \mathrm{~h}$. Cell lysates were analyzed by immunoblotting as indicated. (C) L929 cells were treated with TZ for 2, 4 and $6 \mathrm{~h}$. Cell lysates were analyzed by immunoblotting as indicated. (D) Control-shRNA or MLKL-shRNA HT29 cells were treated with TSZ for $4 \mathrm{~h}$. Conditioned media were collected and analyzed by silver staining. (E) List of cell-surface proteins identified by mass spectrometry in the conditioned medium from TSZ-treated HT29 cells. (F) shRNA-control or shRNA-MLKL HT29 cells were treated with TSZ for $4 \mathrm{~h}$. Cell lysates were analyzed by immunoblotting with antibodies against EpCAM, BCAM, TfR, MLKL and actin. Data shown are representative of three independent experiments.

4A). Remarkably, when the kinase-dead RIP3 was co-expressed with MLKL, or when the two main RIP3-phosphorylation sites of MLKL were mutated, MLKL failed to be pulled down by immunoprecipitating ADAM10, suggesting RIP3-mediated phosphorylation of MLKL is critical for the interaction of MLKL with ADAM10 (Figure 4B). Importantly, the phosphorylated MLKL, not RIP3, formed a complex with ADAM10 under necro- 
A

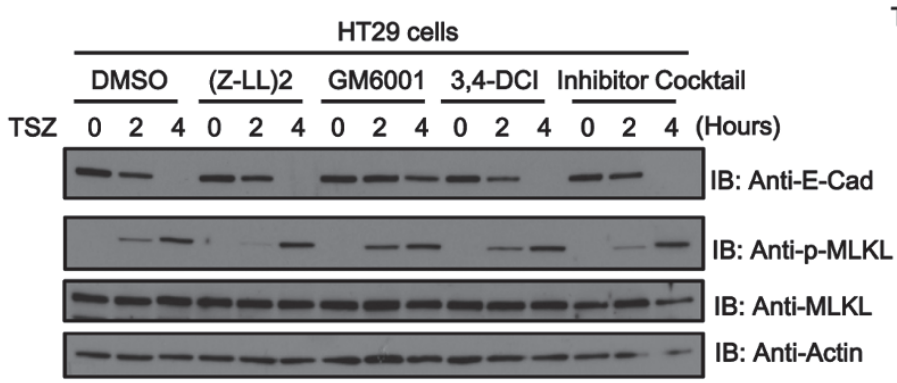

C

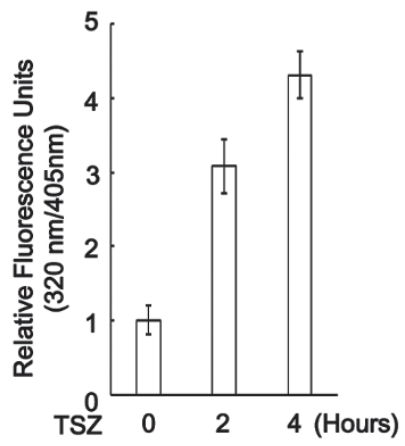

D

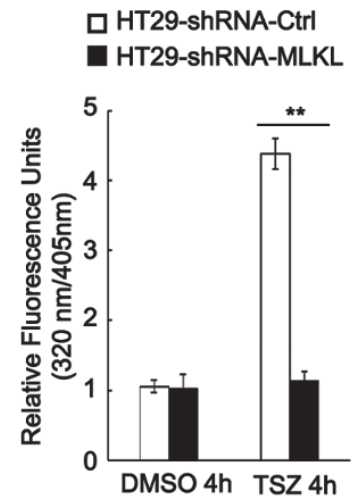

F

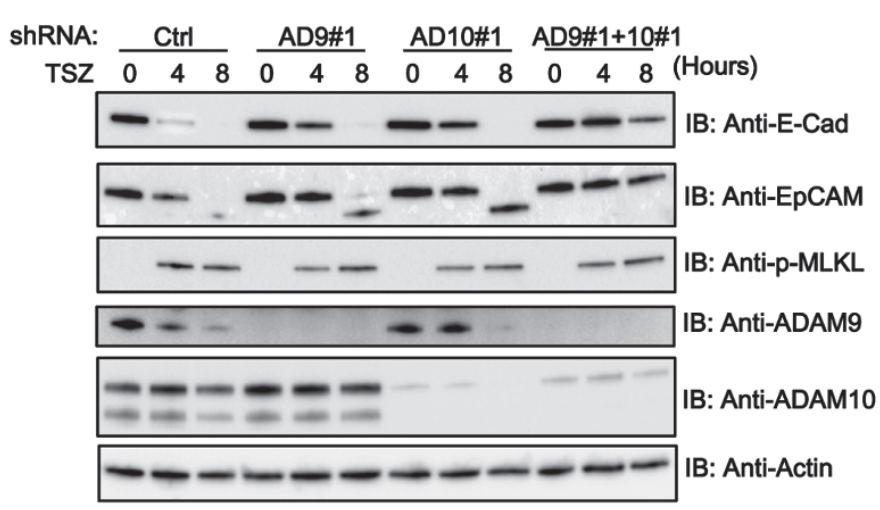

B

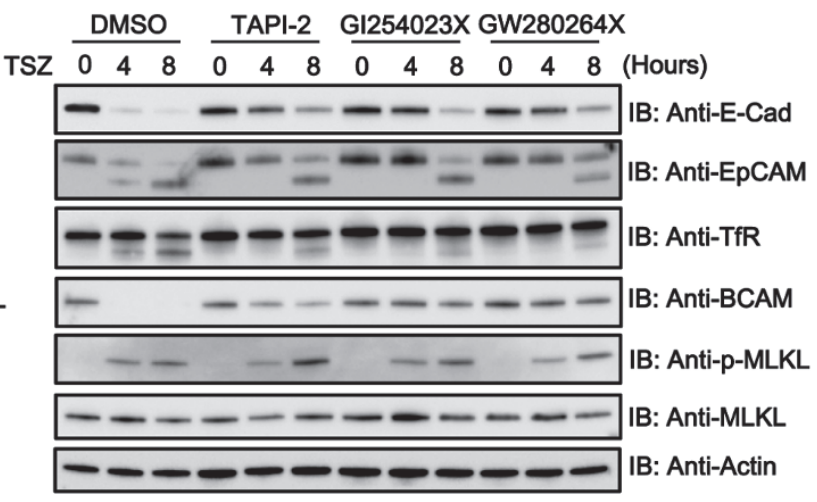

E

shRNA: Ctrl AD9\#1 AD10\#1 AD15\#1 AD17\#1

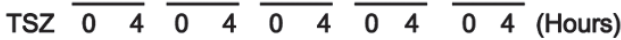

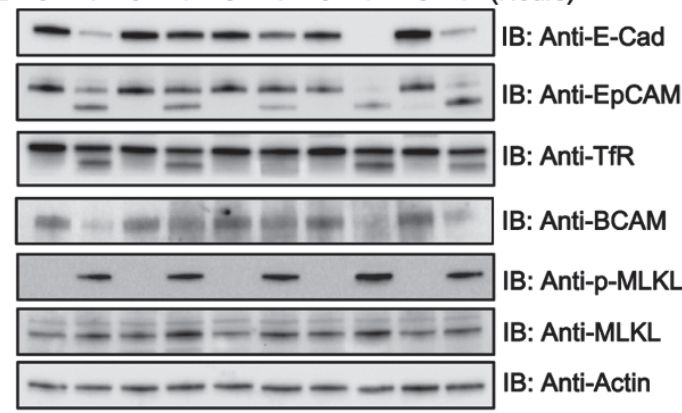

G

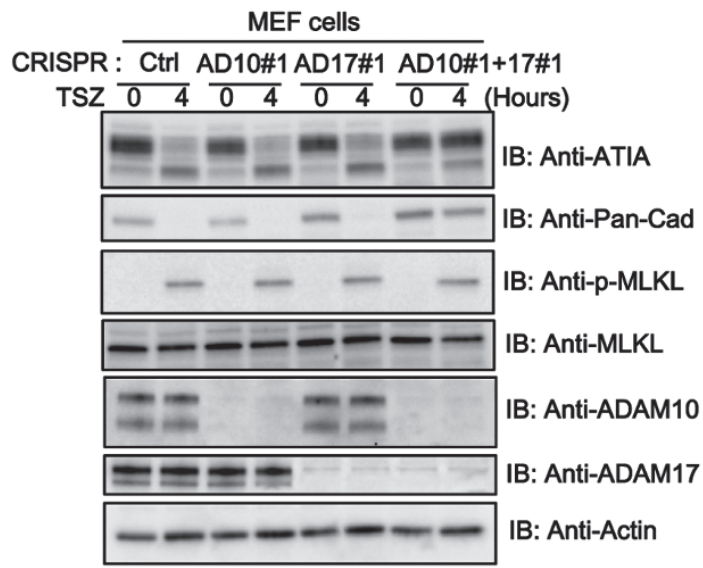

Figure 3 Activation of ADAM metalloproteinases mediates shedding of cell-surface proteins in necroptosis. (A) HT29 cells were treated with TSZ in the presence of different protinase inhibitors for the indicated time points. Cell lysates were analyzed by immunoblotting as indicated. (B) HT29 cells were treated with TSZ in the presence of ADAM metalloproteinase inhibitors TAPI-2 $(10 \mu \mathrm{M}), \mathrm{GI} 254023 \mathrm{X}(10 \mu \mathrm{M})$, GW280264X $(10 \mu \mathrm{M})$, or DMSO control $(0.1 \%)$ at different time points. Cell lysates were analyzed by immunoblotting with the indicated antibodies. (C) Cell-associated metalloprotease activity measured in HT29 cells treated with TSZ at indicated time points. (D) shRNA-Control or shRNA-MLKL HT29 cells were treated with TSZ for $4 \mathrm{~h}$ and cell-associated metalloprotease activity was measured. Results shown are averages \pm SEM. from three independent experiments. (E) HT29 cells expressing shRNA targeting ADAM9\#1, 10\#1, $15 \# 1$ and 17\#1 were treated with TSZ for $4 \mathrm{~h}$. Cell lysates were analyzed by immunoblotting with the indicated antibodies. (F) shRNA control, ADAM9\#1, ADAM10\#1 or ADAM9\#1 and ADAM10\#1 HT29 cells were treated with TSZ at different time points and the cell lysates were analyzed by immunoblotting with the indicated antibodies. (G) CRISPR control, ADAM10\#1, ADAM17\#1 and ADAM10\#1/17\#1 double-knockout MEF cells were treated with TSZ for $4 \mathrm{~h}$. The cell lysates were analyzed by immunoblotting as indicated. Data shown are representative of three independent experiments. ${ }^{* *} p<0.01$. 
A

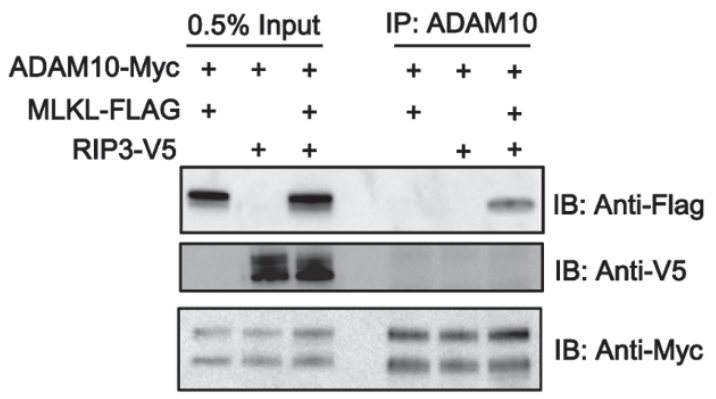

C

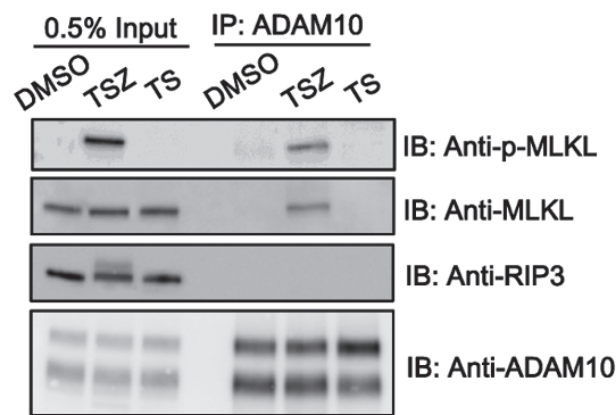

B

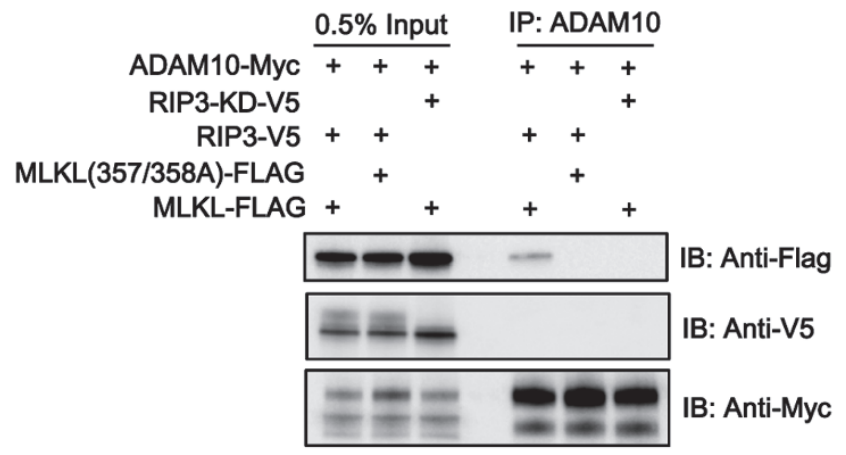

D

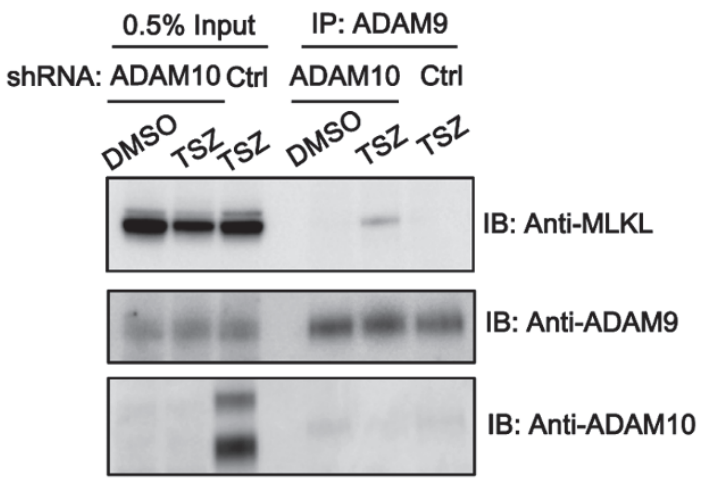

E

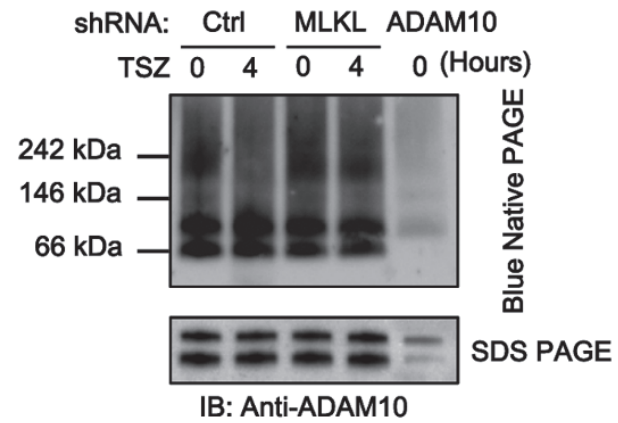

Figure 4 MLKL forms complex with ADAMs to mediate activation of ADAM metalloproteases. (A) HEK293 cells were transfected with ADAM10-Myc and MLKL-FLAG, RIP3-V5 or MLKL plus RIP3-V5 as indicated. Cell lysates were immunoprecipitated with anti-ADAM10 antibody. The immunoprecipitated complexes were analyzed by immunoblotting as indicated. (B) HEK293 cells were transfected with ADAM10-Myc and other constructs as indicated. Cell lysates were immunoprecipitated with anti-ADAM10 antibody. The immunoprecipitated complexes were analyzed by immunoblotting as indicated. (C) HT29 cells were treated with TSZ or TS for $4 \mathrm{~h}$. Cell lysates were immunoprecipitated with anti-ADAM10 antibody. The immunoprecipitated complexes were analyzed by immunoblotting as indicated. (D) shRNA-Control or shRNA-MLKL HT29 cells were treated with TSZ for $4 \mathrm{~h}$. Cell lysates were immunoprecipitated with anti-ADAM9 antibody. (E) shRNA-control or -MLKL HT29 cells were treated with TSZ for $4 \mathrm{~h}$. The cells were lysed under native condition and analyzed by blue native PAGE (upper panel) or SDS-PAGE (lower panel) with anti-ADAM10 immunoblotting. The cell lysate from ADAM10 knockdown cells was used as negative control for ADAM10 antibody immunoblotting. Data shown are representative of three independent experiments. 
ptotic (TSZ), but not apoptotic (TS), condition in HT29 cells (Figure 4C). Moreover, MLKL only complexed with ADAM9 in ADAM10 shRNA knockdown cells (Figure 4D), suggesting a possible redundant function of ADAM9 and ADAM10 in mediating the cleavage of cell-surface proteins [26]. Previous studies suggested that the activation of ADAM10/17 is regulated by the conformational change from oligomer to monomer on cell surface $[27,28]$. We then performed a blue native PAGE to determine the oligomeric status of ADAM10 during necroptosis. Interestingly, we found that TSZ treatment decreased the abundance of ADAM10 oligomers in HT29 cells but not in MLKL knockdown cells (Figure $4 \mathrm{E})$. Therefore, these data suggest that the phosphorylated MLKL may mediate the activation of ADAMs through forming a complex with ADAMs.

\section{Activation of ADAM metalloproteases accelerates necro-} ptotic cell death and promotes inflammation

Importantly, knockdown of ADAM9 or ADAM10 and particularly, ADAM9 and ADAM10 double knockdown significantly protected cells from TSZ-, but not TS-, induced cell death (Figure 5A and Supplementary information, Figure S4A). Moreover, the disruption of cell-cell contact and cell attachment by the onset of necroptosis was significantly slowed in ADAM9 and ADAM10 double knockdown cells compared with the control cells (Figure 5B). Similar results were obtained in ADAM10 and ADAM17 double knockout MEFs and L929 cells (Supplementary information, Figure S4B and $\mathrm{S} 4 \mathrm{C})$. In addition, the specific ADAM metalloproteinase inhibitor GW280264X also significantly delayed cell death in HT29 cells (Supplementary information, Figure S4D and S4E). We previously found that calcium influx is an early event of necroptosis [10]. However, calcium depletion only protected cells from plasma membrane permeabilization, but not from the loss of cell-cell contact (Supplementary information, Figure S4D and S4E). Furthermore, calcium depletion did not prevent TSZ-induced cleavage of E-cadherin (Supplementary information, Figure S4F). These data suggest that the activation of ADAMs and calcium influx are two separate events at the early stage of necroptosis. Interestingly, we found that although the ADAM metalloprotease activity was increased when HT29 cells or MEFs were cultured in suspension, knockdown of ADAMs had limited effect on TSZ-induced necroptosis (Supplementary information, Figure S5A-S5C) and similarly, knockdown of ADAM9 and ADAM10 in Jurkat ${ }^{\mathrm{FDD}^{-/-}}$cells did not protect against TSZ-induced necroptosis either (Supplementary information, Figure S5D). Moreover, we found that HT29 cells tended to form aggregates when cultured at higher den- sity $\left(5 \times 10^{5}\right.$ cell $\left./ \mathrm{ml}\right)$ and knockdown of ADAM9/10 did not affect necroptosis when these cells formed aggregates (Supplementary information, Figure S5E), suggesting that cell aggregation is different from cell adhesion and does not affect the process of necroptosis. Collectively, our data indicate that ADAM activation promotes necroptosis specifically in adherent cells.

It is known that ADAM-mediated shedding of cell-surface molecules promotes inflammation [29]. To examine whether ADAM activation in necroptotic cells plays a role in necroptosis-triggered inflammatory responses, we first tested the effect of cell culture medium on macrophage activation in vitro and found that the medium of TSZ-treated wild-type, but not ADAM10/17 double knockout, MEFs induced much greater upregulation of IFN- $\gamma$, IL-6, TNF- $\alpha$ and VEGF expression in J774 macrophage cells (Supplementary information, Figure S6A and S6B). The protein levels of IL-6 and TNF- $\alpha$ in the supernatant of $\mathrm{J} 774$ cells was much lower in the knockout cells as well (Supplementary information, Figure $\mathrm{S6C}$ ). These data indicate a possible role of ADAMs in necroptosis-induced inflammatory responses.

To test this possibility in vivo, we intraperitoneally injected TSZ-treated control or ADAM10/17 double knockout MEFs, cultured in suspension, into C57BL/6 mice. Sixteen hours after injection, mice injected with ADAM10/17 double knockout MEFs had much less neutrophils in their peritoneal cavity compared with mice injected with control cells, suggesting that the inflammatory responses triggered by ADAM10/17 double knockout cells were significantly lower [30, 31] (Figure $5 \mathrm{C}$ and $5 \mathrm{D})$. To further evaluate the role of ADAM activation in necroptosis/inflammation in vivo, we analyzed the effect of ADAM inhibition in caerulein-induced pancreatitis mouse model and found that ADAM inhibition with GW280264X dramatically protected mice from pancreas acinar cell loss and necrosis (Figure 5E). GW280264X-treated mice had significantly lower levels of serum amylase in response to caerulein (Figure 5F). These data further support that the activation of ADAMs promotes cell death and inflammation.

\section{Necroptosis promotes cell migration and invasion}

While the medium of necroptotic cells triggered inflammatory responses of macrophages, it did not alter cell proliferation and viability of HT29, A549 and MDAMB-231 cells (Figure 6A). However, the medium of TSZ-treated HT29 cells significantly promoted A549 and MDA-MB-231 cell migration by transwell migration assay (Figure 6B). The conditioned medium of TSZ-treated cells also increased cell invasion as determined by transwell matrigel invasion assay (Figure 6C). Importantly, 
A

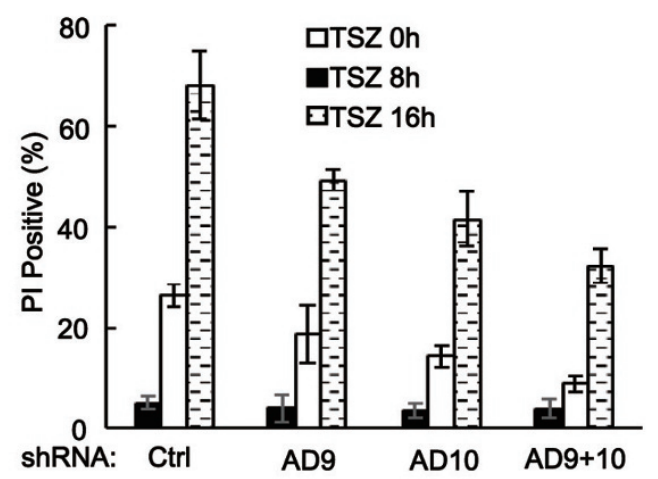

B

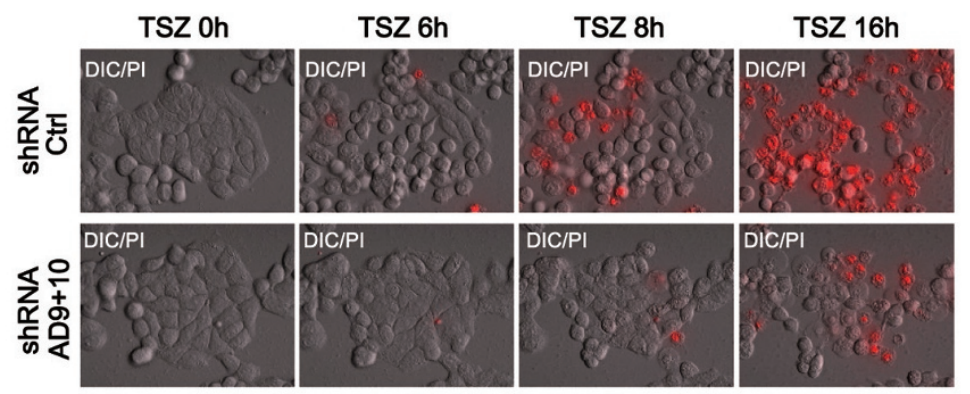

C

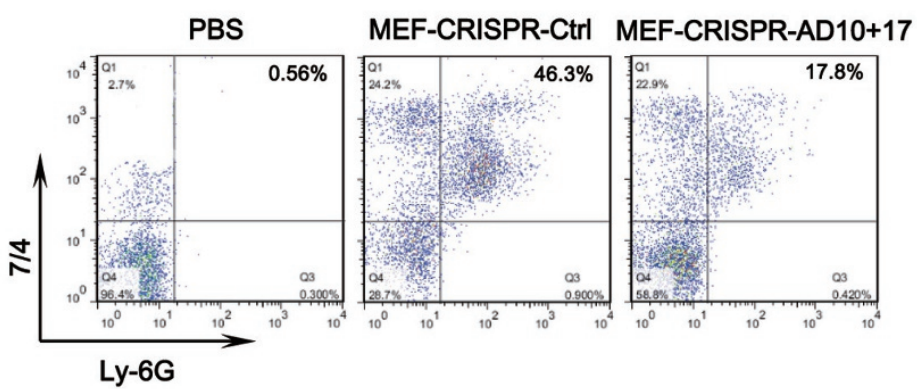

D

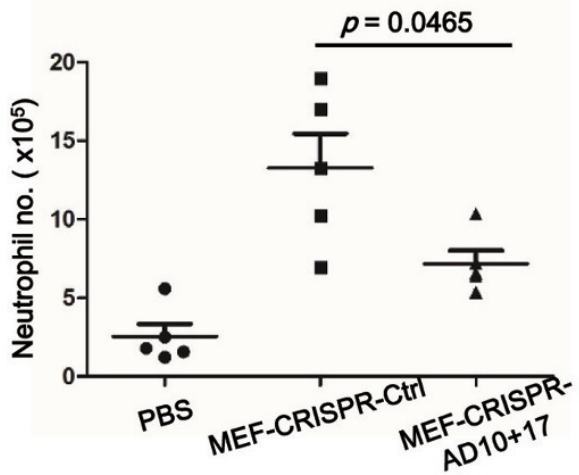

E

PBS

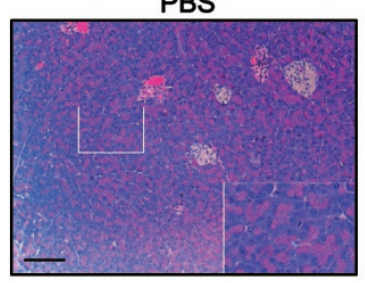

Caerulein

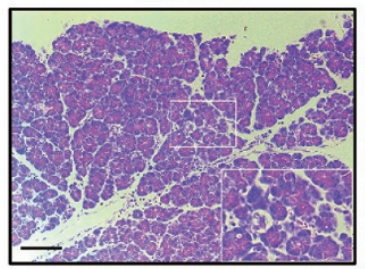

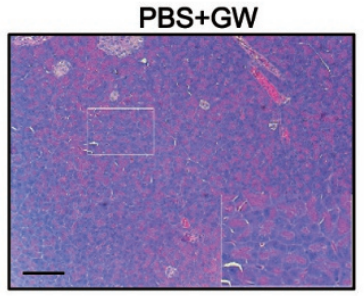

Caerulein+GW

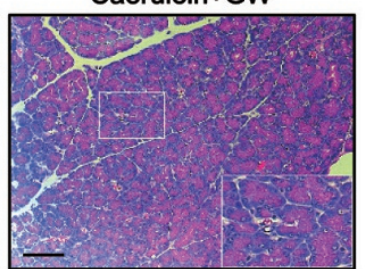

F

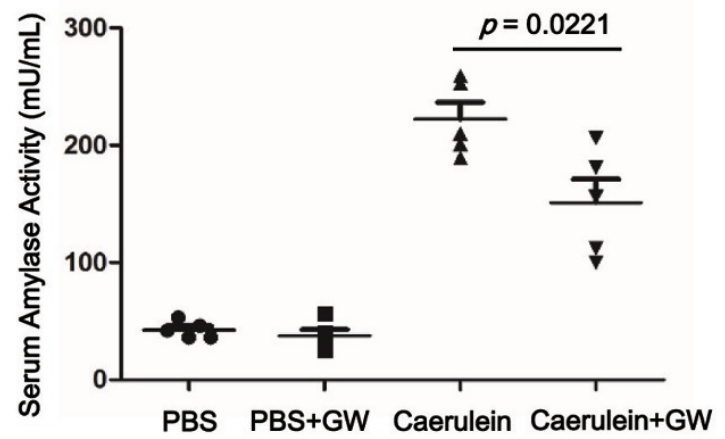

Figure 5 Activation of ADAM metalloproteases accelerates necroptotic cell death and promotes inflammation. (A) HT29 cells expressing shRNAs targeting ADAM9, ADAM10 or ADAM9 and ADAM10 were treated with TSZ at different time points and cell death was determined by PI staining. Results shown are averages \pm SEM from three independent experiments. (B) Timelapse microscopic analysis of cell morphologic changes after induction of necroptosis by TSZ in shRNA-control or shRNA -ADAM9/10 HT29 cells. (C) Representative dot plots of Ly-6G and 7/4 expression on peritoneal cavity cells and (D) neutrophil influx numbers in peritoneal cavity in C57BL/6 mice injected with PBS, necroptotic CRISPR-control- or CRISPR-ADAM10/17 MEF cells. (E) Mice were pre-treated with DMSO vehicle or GW280264X and followed by induction of acute pancreatitis by caerulein. Representative H\&E-stained pancreatic sections from PBS or caerulein-treated mice were shown. (F) Serum amylase activity was measured in each group of mice ( $n=5$ per group). 
A

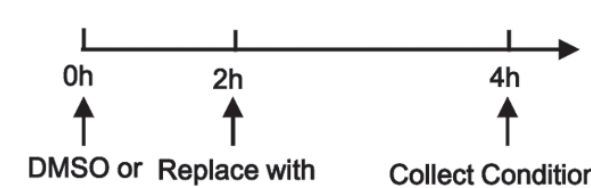

Collect Conditioned

口DMEM

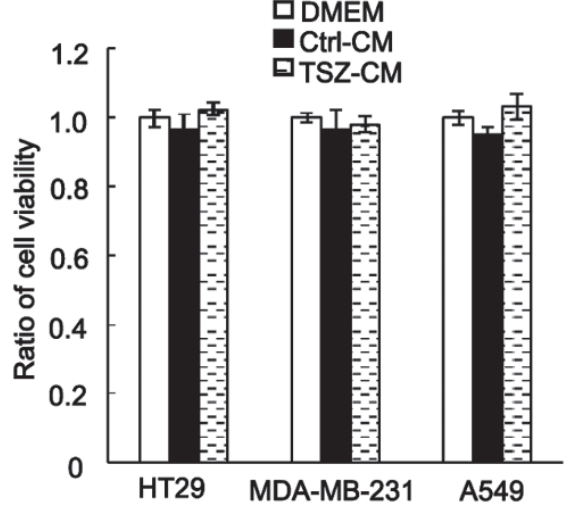

C
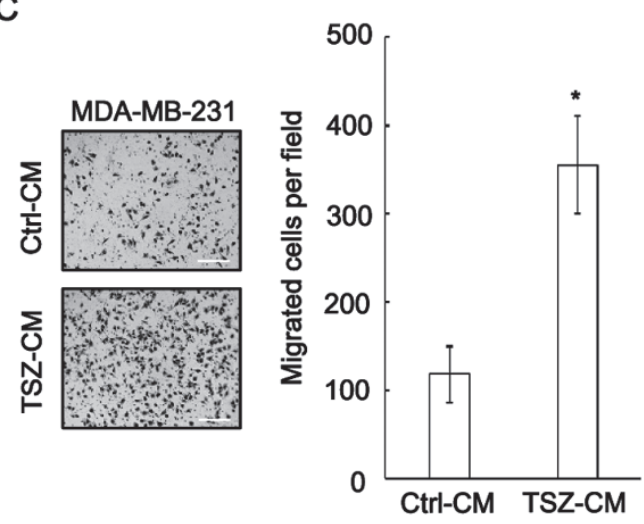

E

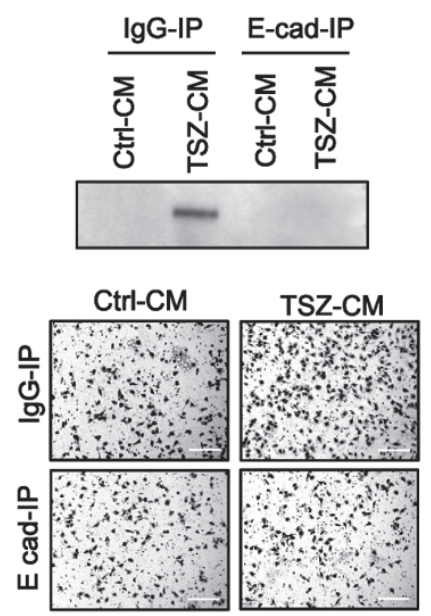

B
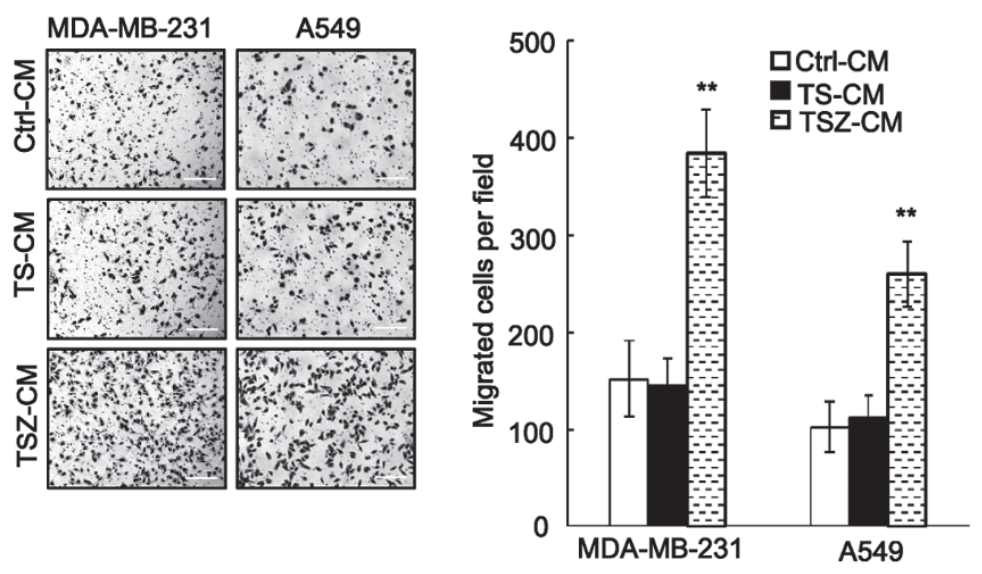

D
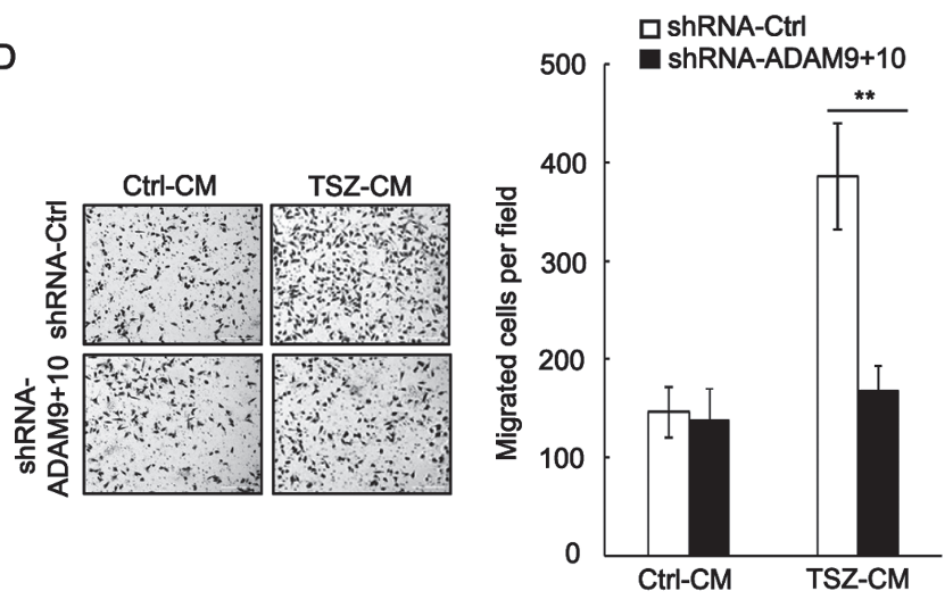

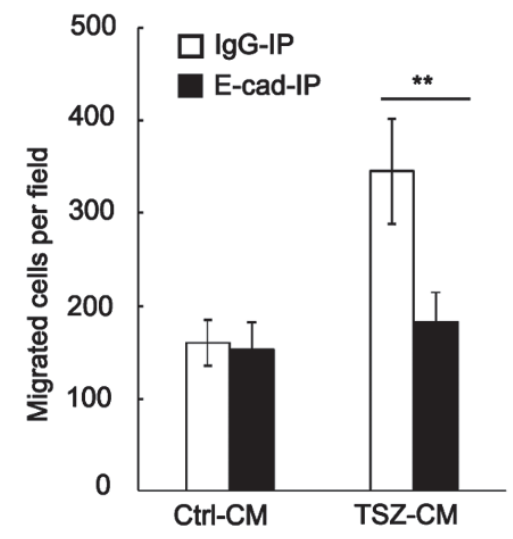


Figure 6 Necroptosis promotes cell migration and invasion. (A) Upper diagram, flow chart of isolation of conditioned medium from DMSO-treated (Ctrl-CM) or TSZ-treated (TSZ-CM) HT29 cells. Lower graph, HT29, MDA-MB-231 and A549 cells were treated with DMEM, Ctrl-CM or TSZ-CM for $48 \mathrm{~h}$, respectively. Relative cell viability was analyzed by MTT assay. Results shown are averages \pm SEM. (B) MDA-MB-231 and A549 cells were treated with Ctrl-CM, TS-CM or TSZ-CM for $12 \mathrm{~h}$. Cell migration was analyzed by transwell assay. Left, representative images of transwell assay. Right, quantitative data of migrated cells. (C) MDA-MB-231 cells were seeded in transwells covered with $10 \%$ Matrigel gel and treated with Ctrl-CM or TSZ-CM from HT29 cells for $24 \mathrm{~h}$. Left, representative images of transwell assay. Right, quantitative data of migrated cells. (D) MDAMB-231 cells were treated with the conditioned media from control-shRNA or ADAM9/10-shRNA HT29 cells for $12 \mathrm{~h}$. Cell migration was analyzed by transwell assay. Left, representative images of transwell assay. Right, quantitative data of migrated cells. (E) Ctrl-CM or TSZ-CM were immunoprecipitated with IgG-control (IgG-IP) or E-cadherin antibody (E-cad-IP) and then used to treat MDA-MB-231 cells for $12 \mathrm{~h}$. Cell migration was analyzed by tranwell assay. Upper-left panel, immunoblotting analysis of the levels of soluble E-cadherin in conditioned medium after immunoprecipitation. Lower-left panel, representative images of transwell assay. Right panel, quantitative data of migrated cells. Results shown are averages \pm SEM. ${ }^{* *} p<0.01$; Scale bar, $200 \mu \mathrm{m}$. All data were representative results of three independent experiments.

the conditioned medium of ADAM9/10 knockdown cells did not stimulate cell migration (Figure 6D). Since previous studies showed that soluble E-cadherin promoted cell migration and invasion [23, 32], we then depleted the cleaved E-cadherin from the medium of TSZ-treated cells and found that removing E-cadherin abolished the promoting effect of the medium on cell migration (Figure 6E). Moreover, we found that the conditioned medium collected from TSZ-treated wild-type, but not ADAM9/10 double knockout, cells promoted the migration of $\mathrm{T}$ cells and monocytes (Supplementary information, Figure S7B and S7C). Therefore, our results suggest that the soluble factors from ADAM-mediated shedding of cell-surface proteins of necroptotic cells promote cell migration and invasion.

\section{Discussion}

Although translocation of active MLKL to the plasma membrane is an essential step in necroptosis, the execution mechanism of necroptosis is unclear. While phosphorylated MLKL may promote necroptosis through activating plasma membrane ion channels or exerting its own pore-forming activity, these functions of MLKL are either indirect or relatively late in the death execution process. In addition, it is commonly assumed that protease activation is not necessary for the engagement of necroptosis and often this assumption is used as a hallmark to distinguish necroptosis from caspase-dependent apoptosis. Our current findings suggest that ADAM metalloprotease activation not only occurs at the early stage of necroptosis, but also plays a key role in promoting necroptosis in adherent cells.

Activation of ADAMs has been reported in the cells attacked by some pore-forming bacterial proteins [33]. Particularly, it has been demonstrated that the staphylococcal $\alpha$-toxin binds to ADAM10 and renders cells more susceptible to attack by low toxin concentration
$[24,34]$. Our study suggest that cell adhesion disruption by the activation of ADAMs plays an important role in necroptotic cell death. Although the detailed mechanism of ADAM activation is still poorly understood, a recent study reported that ADAM10 and ADAM17 form homodimers on the cell surface and the transition of ADAM10 or 17 homodimers to monomers is critical for metalloprotease activation [27]. We show that MLKL forms a complex with multiple ADAMs upon necroptotic stimulus. Moreover, necroptotic stimulus decreases the abundance of ADAM10 oligomers in HT29 cells through a MLKL-dependent mechanism, suggesting MLKL may activate ADAM10 by inducing a conformational change of the protease during necroptosis.

In vertebrate cells, ADAMs mediate the release of diverse membrane-associated proteins including cadherins, Fas ligand, TNF- $\alpha$, ErbB2, EpCAM and many others [35]. The shedding process induced by activation of ADAMs modulates cell-cell contacts, cell-matrix adhesion, cell migration and inflammation. Accordingly, dysregulation of ADAMs is related to several pathologies such as autoimmune diseases, neurodegeneration, infection, inflammation and cancer [25]. It is generally believed that necroptosis directly triggers inflammation by the release of cellular components described as damage-associated molecular patterns (DAMPs) from permeabilized cells [36]. Although this consensus notion is supported by the observation that DAMPs are released from necroptotic cells in vitro, there is currently no in vivo evidence to prove that the release of DAMPs indeed triggers inflammation, which may be due to the redundancy among DAMPs [36]. Our study demonstrates that necroptosis not only can result in the release of DAMPs from permeabilized cells but also induces shedding of diverse cell-surface proteins by activating ADAMs when the cell plasma membrane is still intact. Currently, there are more than 100 cell-surface proteins that are identified as substrates of ADAM metalloproteases [35]. Besides 
adhesion proteins (e.g., E cadherin and EpCAM), ADAMs also cleave other factors including cytokines (e.g., TNF- $\alpha$, Fas ligand) and cytokine receptors (e.g., IL-6R and TNF-R), which are pro-inflammatory [35]. Thus, our study reveals a previously unknown mechanism of how necroptosis directly induces inflammation. Moreover, in vivo studies have suggested that necroptotic cell death can induce inflammation indirectly by disrupting the epithelial barrier to trigger microbe-driven immune responses in the intestinal epithelium [37]. Our results demonstrate that the necroptotic stimulus induces proteolysis of E-cadherin and cell-cell contact can be attenuated by knocking down of ADAM9/10, implicating that activation of ADAMs during necroptosis may further contribute to the disruption of the epithelial barrier and exacerbate inflammation in vivo. Our study also demonstrates that proteolytic cleavage and the release of E-cadherin fragments from necroptotic cells promotes tumor cell migration and invasion. Since elevated serum soluble E-cadherin was reported in a wide variety of cancer types and promotes tumor growth and metastasis [32], it would be interesting to investigate the role of necroptosis in tumor metastasis in the future.

Collectively, our findings reveal a novel mechanism of necroptosis-induced inflammation and cell migration and provide new insight about how necroptosis may modulate immune response or cause tissue damage, deepening our understanding of the physiological and pathological functions of necroptosis.

\section{Materials and Methods}

\section{Constructs, shRNAs, reagents and antibodies}

C-terminal FLAG epitope-tagged human MLKL was generated by subcloning MLKL into pcDNA3.1-FLAG from pCMV-Tag2A-MLKL, which was described previously [10]. MLKL point mutants were generated using site-directed mutagenesis and confirmed by sequencing analysis. pcDNA3.1/V5-RIP3 and pcDNA3.1/V5-RIP3-D160N were generated by subcloning RIP3 into pcDNA3.1/V5 from RIP3- and RIP3-D160N-EGFP constructs, which were kindly provided by Dr Francis Ka Ming Chan (University of Massachusetts Medical School). E-cadherin-GFP was a gift from Dr Jennifer Stow (Addgene \# 28009). pRK5M-ADAM10 was a gift from Dr Rik Derynck (Addgene \# 31717). ORIA-1-YFP was a gift from Dr Anjana Rao (Addgene \# 19756). All plasmid constructs were confirmed by DNA sequencing.

shRNA against MLKL was described previously [10]. The lentivirus silencing vector expressing shRNA targeting human ADAM9\#1 (TRC number: TRCN0000046978), ADAM9\#2 (TRC number: TRCN0000046980), ADAM10\#1 (TRC number: TRCN0000006675), ADAM10\#2 (TRC number: TRCN0000006674), ADAM15\#1 (TRC number: TRCN0000371222) and ADAM17\#1 (TRC number: TRCN0000052172) were obtained from Sigma.

For generation of CRISPR-Cas9 lentivirus constructs, two guide oligonucleotides against mouse ADAM10 corresponding to the coding sequence 5'-AGACTGCTCGTTTGGCACGC-3' (ADAM10\#1) and the coding sequence 5'-CTCATGTGAGACTGCTCGTT-3' (ADAM10\#2); two guide oligonucleotides against mouse ADAM17 corresponding to the coding sequence 5'-CACTTTGGTGCCTTTCGTCC-3' (ADAM17\#1) and the coding sequence 5'-GTGCTGCTGAATATTAGCTA-3' (ADAM17\#2) were cloned into the Cas9-containing lentiCRISPR-v2 vector, which was a gift from Dr Feng Zhang (Addgene \#52961).

TNF- $\alpha$ and $z-V A D-f m k$ were purchased from R\&D. Cycloheximide, GM6001, 3,4-DCI, TAPI-2, GI254023 and protease inhibitor cocktail were purchased from Sigma. GW280264X was purchased from AOBIOUS (Gloucester, MA, USA). Smac mimetic was a gift from Dr Shaomeng Wang (University of Michigan, Ann Arbor). Mouse Inflammatory Cytokines \& Receptors PCR Array was purchased from QIAGEN. Antibodies were from commercial sources: anti-actin (A3853) and anti-Tubulin (T9026) from Sigma; anti-E-cadherin (HECD1; ab1416), anti-human MLKL (ab184718), anti-human phospho-MLKL (ab187091), anti-mouse phospho-MLKL (ab196436), anti-ADAM10 (ab1997), anti-anti-neutrophil [7/4] (ab105157) and anti-ADAM17 (ab2051) from Abcam. Anti-mouse Ly-6G (11-9668) from eBioscience. Anti-human ectodmain of ADAM9 (AF939), anti-human ectodmain of ADAM10 (MAB1427), anti-human EpCAM (MAB960), anti-human TfR (AF2474) and anti-human BCAM (MAB1481) from R\&D Systems. Anti-E-cadherin C-terminal (4A2C7) from ThermoFisher. Anti-pan-cadherin (28E12) from Cell Signaling Technology). Antibody for mouse MLKL was kindly provided by Dr Jiahuai Han (Xiamen University). Anti-ATIA/vasorin was described previously [21].

\section{Cell culture}

HT29, L929 and MEF cells were cultured in DMEM. Jurkat FADD $^{-/-}$cells were cultured in RPMI 1640. Wild-type and MLKL-deficient MEFs were kindly provided by Dr Jiahuai Han (Xiamen University). All media were supplemented with 10\% FBS (v/v), $2 \mathrm{mM}$ L-glutamine and $100 \mathrm{U} / \mathrm{ml}$ penicillin/streptomycin.

For suspended cell culture, HT29 cells or MEFs were lifted using Cellstripper (Mediatech) and a non-enzymatic cell dissociation solution, in order to avoid degradation of cell-surface proteins. Cells were washed with PBS and cultured with DMEM at density of $5 \times 10^{4}$ cells $/ \mathrm{ml}$ in non-treated 12 -well tissue culture plate. On the second day of culture, cells were treated with TSZ to induce necroptosis.

\section{Lentivirus Infection}

HEK293T cells were co-transfected with pCMV-VSV-G and pCMV-dr8.2-dvpr and shRNA or CRISPR-Cas9 transfer plasmids. After $24 \mathrm{~h}$, supernatant was collected and this lentiviral preparation was used to infect cells. After $24 \mathrm{~h}$ of infection, cells were selected with puromycin for an additional $48 \mathrm{~h}$. For generation of ADAM10 or ADAM17 knockout MEFs, CRISPR knockout cells were grown at low density for 2 weeks to select clones. Protein expression was determined by immunoblotting to identify clones without ADAM10 or ADAM17 expression. The ADAM10 and ADAM17 double knockout MEFs were generated by co-infection of ADAM10 and ADAM17 CRISPR-Cas9 lentiviruses. The double knockout clones were selected and identified by immunoblotting analysis. 


\section{Necroptosis induction}

Necroptosis was induced by pretreatment with z-VAD-fmk (20 $\mu \mathrm{M})$ and Smac mimetic $(10 \mathrm{nM})$ or cycloheximide $(10 \mu \mathrm{g} / \mathrm{ml})$ for $30 \mathrm{~min}$ and followed by TNF- $\alpha(30 \mathrm{ng} / \mathrm{ml})$, or TRAIL $(10 \mathrm{ng} / \mathrm{ml})$ or FasL $(10 \mathrm{ng} / \mathrm{ml})$ for the indicated time periods. Apoptosis was induced by TNF- $\alpha(30 \mathrm{ng} / \mathrm{ml})$ and Smac $(10 \mathrm{nM})$ for the indicated time periods. For protease inhibitors treatment, GM6001 $(10 \mu \mathrm{M})$, (Z-LL)2 Ketone $(10 \mu \mathrm{M}), 3$,4-DCI $(100 \mu \mathrm{M})$, TAPI-2(10 $\mu \mathrm{M})$, GI254023X $(10 \mu \mathrm{M})$, GW280264X $(10 \mu \mathrm{M})$ and Protease Inhibitor Cocktail (1:200) were used to pretreat cells for $1 \mathrm{~h}$ and then treated with TSZ for the indicated time points.

\section{Transwell migration assay}

A transwell migration assay with 6.5-mm-diameter polycarbonate filters ( $8-\mu \mathrm{m}$ pore size) was used. For cell invasion assay, the filter of the transwell plate (BD Biosciences) was coated with Matrigel (BD biosciences). The bottom chambers were filled with $500 \mu \mathrm{l}$ of DMEM containing $10 \%$ FBS. Cells $\left(4 \times 10^{4}\right)$ suspended in $200 \mu \mathrm{l}$ of conditioned DMEM containing $0.5 \%$ FBS were seeded in the top chamber. For cell migration assay, cells were allowed to migrate for $12 \mathrm{~h}$. For Matrigel invasion assay, cells were allowed to migrate for $24 \mathrm{~h}$. Non-migrated cells were removed with cotton swabs, and migrated cells were fixed with cold $4 \%$ paraformaldehyde and stained with $1 \%$ crystal violet. Images were taken using an inverted microscope (10× magnification; Olympus), and migrated cells were quantified by manual counting.

\section{Chemotaxis assay}

T-cell and monocytes chemotaxis was assayed using 24-well Transwell chambers with $5 \mu \mathrm{m}$ pores (Corning). A total of $10^{5}$ Jurkat $^{\mathrm{FADD}^{-/-}}$or THP-1 cells in $100 \mu 1$ chemotaxis buffer (RPMI $1640 / 0.5 \%$ BSA) were placed in the upper chambers. Conditioned medium collected from necroptotic cells was placed in the lower wells and the chambers were incubated for $3 \mathrm{~h}$ at $37^{\circ} \mathrm{C}$. Migrated cells located in the bottom wells were collected and counted by FACS. All experiments were conducted in triplicate. The percentage of migration was calculated by the number of cells in the lower chamber divided by the sum of cells put in the upper chamber at the beginning of experiments.

\section{ADAM metalloprotease activity measurement}

Cells $\left(3 \times 10^{4}\right)$ were seeded in 96 -well plate overnight and then treated with DMSO or TSZ for the indicated times. Cells were washed with $25 \mathrm{mM}$ Tris, $\mathrm{pH} 8.0$ buffer and incubated for $30 \mathrm{~min}$ at $37^{\circ} \mathrm{C}$ with $10 \mu \mathrm{M}$ fluorogenic peptide substrate (Mca-PLAQAVDpa-RSSSR-NH2, R\&D Systems) diluted in $25 \mathrm{mM}$ Tris, $\mathrm{pH}$ 8.0. Fluorescence intensity was read on a Molecular Devices SpectraMax instrument and analyzed by SoftMaxPro software.

\section{Real-time PCR analysis}

RNA was extracted using TRIzol (ThermoFisher) and quantified by spectrophotometry (NanoDrop, Peqlab). RNA was reverse-transcribed using Superscript III reverse transcriptase (ThermoFisher), according to the manufacturer's protocol. Regular PCRs were performed using MyTaq Red Mix (Bioline). Real-time PCRs were performed using SensiFAST SYBR Hi-ROX Kit (Bioline). The sequences of oligonucleotides used in RT-PCR are listed in Supplementary information, Table S1. For regular PCR analysis, the PCR products were subjected to electrophoresis in $1.5 \%$ agarose gels, visualized under UV light after ethidium bromide staining, and then imaged. For real-time PCR, the threshold cycle number for ADAMs was normalized to that of GAPDH, and the resulting value was converted to a linear scale. All assays were performed at least three times from independent RNA preparations.

\section{Immunoblotting, cross-linking and immunoprecipitation}

Cells were collected and lysed in M2 buffer (20 mM Tris, $\mathrm{pH}$ 7, 0.5\% NP40, $250 \mathrm{mM} \mathrm{NaCl}, 3 \mathrm{mM}$ EDTA, 3 mM EGTA, $2 \mathrm{mM}$ DTT, $0.5 \mathrm{mM}$ PMSF, $20 \mathrm{mM}$-glycerol phosphate, $1 \mathrm{mM}$ sodium vanadate, $1 \mu \mathrm{g} / \mathrm{ml}$ Leupeptin). Cell lysates were separated by SDSPAGE and analyzed by immunoblotting. The dilution ratio of the antibodies used for western blotting is 1:1 000. The proteins were visualized by enhanced chemiluminescence, according to the manufacturer's instruction (Amersham) .

For endogenous immunoprecipitation, cross-linking of cellular proteins was performed before cell lysis. Cells were washed three times with ice-cold PBS solution and incubated with cross-linking reagent (DSP, $2 \mathrm{mM}$ ) in PBS for $30 \mathrm{~min}$ at room temperature, followed by incubation of $10 \mathrm{mM}$ Tris, $\mathrm{pH} 7.5$ buffer for $15 \mathrm{~min}$ to quench the reaction. Then, cells were lysed in M2 buffer containing $1 \%$ digitonin. The samples were precipitated with antibody $(1 \mathrm{~g})$ and protein $\mathrm{G}$-agarose beads by incubating at $4{ }^{\circ} \mathrm{C}$ overnight. Beads were washed four to six times with $1 \mathrm{ml} \mathrm{M} 2$ buffer, and the bound proteins were removed by boiling in SDS buffer and resolved in 4\%-20\% SDS-polyacrylamide gels for western blotting analysis.

\section{Mass spectrometry analysis}

HT29 cells $\left(5 \times 10^{7}\right)$ were treated with DMSO or TSZ in $10 \mathrm{ml}$ serum-free DMEM for $4 \mathrm{~h}$. The conditioned medium was collected and ultra-centrifuged at $100000 \times g$ for $1 \mathrm{~h}$ to remove microvesicles. Proteins in the conditioned medium $(5 \mathrm{ml})$ were concentrated by TCA precipitation. Concentrated protein samples were analyzed by SDS-PAGE and in-gel digestions were carried out as previously described [38]. The digested samples were analyzed by nanoLC-MS/MS (nanoACQUITY UPLC and SYNAPT G2 HD mass spectrometer; Waters). Mass spectrometry data were acquired with Data Dependent Analysis mode, processed with PLGS 2.4 software (Waters), and the resulting peak list was searched against the NCBI database with the MASCOT search engine, de novo sequencing was performed using Masslynx Pepseq 4.1 software (Waters). The released proteins in the TSZ-treated conditioned medium were profiled by using DMSO-treated condition as a baseline. The cell-surface proteins were identified by functional annotation from the profiled data.

\section{Necroptotic cells induced in vivo peritonitis}

CRISPR control or ADAM10/17 knockout MEFs were cultured in suspension and pre-treated with TSZ for $2 \mathrm{~h}$ and then washed three times with PBS. Cells were re-suspended in PBS at a density of $1 \times 10^{8}$ cells $/ \mathrm{ml}$. A total of $2 \times 10^{7}$ pre-necroptotic MEFs in 200 $\mu 1$ PBS were intraperitoneally injected into C57BL/6 mouse. PBS alone injection served as control. $16 \mathrm{~h}$ post-injection, peritoneal cavities were washed with $6 \mathrm{ml}$ PBS containing $3 \mathrm{mM}$ EDTA and $10 \mathrm{U} / \mathrm{ml}$ heparin. The total number of peritoneal exudate cells were counted using a hematocytometer and then centrifuged at $450 \times \mathrm{g}$ 
for $10 \mathrm{~min}$. The cell pellets suspended in PBS were double stained with Ly6G and 7/4 antibodies and analyzed by flow cytometry as described [30]. Neutrophil numbers in the peritoneal exudate were determined by multiplying the total peritoneal exudate cell numbers by the percentage of $\mathrm{Ly}-6 \mathrm{G}^{+} 7 / 4^{+}$cells.

\section{Caerulein-induced acute pancreatitis}

C57BL/6 mice at 6-8 weeks of age $(n=5)$ were intraperitoneally injected with GW280264X $(100 \mu \mathrm{g} / \mathrm{kg}$, dissolved in DMSO, final concentration $0.06 \%$ ) or vehicle control $(0.06 \%$ DMSO) for $1 \mathrm{~h}$. Then, mice were intraperitoneally injected with caerulein $(50$ $\mathrm{g} / \mathrm{kg}$, Sigma) every hour for 10 consecutive hours. After the last caerulein injection, mice were re-injected with GW280264X or vehicle control as described above. Mice were killed $16 \mathrm{~h}$ after the last injection and samples of pancreatic tissue and blood were rapidly harvested. Quantification of pancreas injury was performed on pancreatic tissue sections stained with $H \& E$ and serum amylase activity was measured.

\section{Statistical analysis}

The Student's $t$-test and one-way analysis of variance were used for comparison among all different groups. $P<0.05$ was considered statistically significant.

\section{Acknowledgments}

We thank J Han for MLKL-deficient MEF cells and MLKL antibody. This research was supported by the Intramural Research Program of the Center for Cancer Research, National Cancer Institute, National Institutes of Health.

\section{Author Contributions}

ZC and Z-GL conceived the study. ZC did most of the experimental work. AL did some immunoprecipitation and cell death experiments. WL, TL and X-MZ did mass spectrometry experiments. ZC, SC and Z-GL wrote the paper. Z-GL supervised the study. All authors discussed the results and implications and commented on the manuscript at all stages.

\section{Competing Financial Interests}

The authors declare no competing financial interests

\section{References}

1 Zong WX, Thompson CB. Necrotic death as a cell fate. Genes Dev 2006; 20:1-15.

2 Chan FK, Luz NF, Moriwaki K. Programmed necrosis in the cross talk of cell death and inflammation. Annu Rev Immunol 2015; 33:79-106.

3 Zhang DW, Shao J, Lin J, et al. RIP3, an energy metabolism regulator that switches TNF-induced cell death from apoptosis to necrosis. Science 2009; 325:332-336.

4 Sun L, Wang H, Wang Z, et al. Mixed lineage kinase domain-like protein mediates necrosis signaling downstream of RIP3 kinase. Cell 2012; 148:213-227.

5 Zhao J, Jitkaew S, Cai Z, et al. Mixed lineage kinase domain-like is a key receptor interacting protein 3 downstream component of TNF-induced necrosis. Proc Natl Acad Sci USA
2012; 109:5322-5327.

6 Holler N, Zaru R, Micheau O, et al. Fas triggers an alternative, caspase-8-independent cell death pathway using the kinase RIP as effector molecule. Nat Immunol 2000; 1:489-495.

7 Lin Y, Choksi S, Shen HM, et al. Tumor necrosis factor-induced nonapoptotic cell death requires receptor-interacting protein-mediated cellular reactive oxygen species accumulation. J Biol Chem 2004; 279:10822-10828.

8 Cho YS, Challa S, Moquin D, et al. Phosphorylation-driven assembly of the RIP1-RIP3 complex regulates programmed necrosis and virus-induced inflammation. Cell 2009; 137:1112-1123.

9 He S, Wang L, Miao L, et al. Receptor interacting protein kinase-3 determines cellular necrotic response to TNF- $\alpha$. Cell 2009; 137:1100-1111.

10 Cai Z, Jitkaew S, Zhao J, et al. Plasma membrane translocation of trimerized MLKL protein is required for TNF-induced necroptosis. Nat Cell Biol 2014; 16:55-65.

11 Chen X, Li W, Ren J, et al. Translocation of mixed lineage kinase domain-like protein to plasma membrane leads to necrotic cell death. Cell Res 2014; 24:105-121.

12 Wang H, Sun L, Su L, et al. Mixed lineage kinase domain-like protein MLKL causes necrotic membrane disruption upon phosphorylation by RIP3. Mol Cell 2014; 54:133-146.

13 Dondelinger Y, Declercq W, Montessuit S, et al. MLKL compromises plasma membrane integrity by binding to phosphatidylinositol phosphates. Cell Rep 2014; 7:971-981.

14 Vanden Berghe T, Linkermann A, Jouan-Lanhouet S, Walczak $\mathrm{H}$, Vandenabeele P. Regulated necrosis: the expanding network of non-apoptotic cell death pathways. Nat Rev Mol Cell Biol 2014; 15:135-147.

15 Harris TJ, Tepass U. Adherens junctions: from molecules to morphogenesis. Nat Rev Mol Cell Biol 2010; 11:502-514.

16 Degterev A, Huang Z, Boyce M, et al. Chemical inhibitor of nonapoptotic cell death with therapeutic potential for ischemic brain injury. Nat Chem Biol 2005; 1:112-119.

17 Prakriya M, Feske S, Gwack Y, Srikanth S, Rao A, Hogan PG. Orail is an essential pore subunit of the CRAC channel. Nature 2006; 443:230-233.

18 Niessen CM, Leckband D, Yap AS. Tissue organization by cadherin adhesion molecules: dynamic molecular and cellular mechanisms of morphogenetic regulation. Physiol Rev 2011; 91:691-731.

19 Kawamura-Kodama K, Tsutsui J, Suzuki ST, Kanzaki T, Ozawa $\mathrm{M}$. N-cadherin expressed on malignant $\mathrm{T}$ cell lymphoma cells is functional, and promotes heterotypic adhesion between the lymphoma cells and mesenchymal cells expressing N-cadherin. J Invest Dermatol 1999; 112:62-66.

20 Redmer T, Diecke S, Grigoryan T, Quiroga-Negreira A, Birchmeier W, Besser D. E-cadherin is crucial for embryonic stem cell pluripotency and can replace OCT4 during somatic cell reprogramming. EMBO Rep 2011; 12:720-726.

21 Choksi S, Lin Y, Pobezinskaya Y, et al. A HIF-1 target, ATIA, protects cells from apoptosis by modulating the mitochondrial thioredoxin, TRX2. Mol Cell 2011; 42:597-609.

22 Chidgey MA, Clarke JP, Garrod DR. Expression of full-length desmosomol glycoproteins (desmocollins) is not sufficient to confer strong adhesion on transfected L929 cells. J Invest Dermatol 1996; 106:689-695. 
23 Maretzky T, Reiss K, Ludwig A, et al. ADAM10 mediates E-cadherin shedding and regulates epithelial cell-cell adhesion, migration, and $\beta$-catenin translocation. Proc Natl Acad Sci USA 2005; 102:9182-9187.

24 Inoshima I, Inoshima N, Wilke GA, et al. A Staphylococcus aureus pore-forming toxin subverts the activity of ADAM10 to cause lethal infection in mice. Nat Med 2011; 17:13101314.

25 Seals DF, Courtneidge SA. The ADAMs family of metalloproteases: multidomain proteins with multiple functions. Genes Dev 2003; 17:7-30.

26 Moss ML, Powell G, Miller MA, et al. ADAM9 inhibition increases membrane activity of ADAM10 and controls $\alpha$-secretase processing of amyloid precursor protein. $\mathrm{J} \mathrm{Biol} \mathrm{Chem}$ 2011; 286:40443-40451.

27 Xu P, Liu J, Sakaki-Yumoto M, Derynck R. TACE activation by MAPK-mediated regulation of cell surface dimerization and TIMP3 association. Science Signal 2012; 5:ra34.

28 Deng W, Cho S, Su PC, Berger BW, Li R. Membrane-enabled dimerization of the intrinsically disordered cytoplasmic domain of ADAM10. Proc Natl Acad Sci USA 2014; 111:1598715992.

29 Khokha R, Murthy A, Weiss A. Metalloproteinases and their natural inhibitors in inflammation and immunity. Nat Rev Immunol 2013; 13:649-665.

30 Chen CJ, Kono H, Golenbock D, Reed G, Akira S, Rock KL. Identification of a key pathway required for the sterile inflammatory response triggered by dying cells. Nat Med 2007; 13:851-856.
31 Iyer SS, Pulskens WP, Sadler JJ, et al. Necrotic cells trigger a sterile inflammatory response through the Nlrp3 inflammasome. Proc Natl Acad Sci USA 2009; 106:20388-20393.

32 David JM, Rajasekaran AK. Dishonorable discharge: the oncogenic roles of cleaved E-cadherin fragments. Cancer Res 2012; 72:2917-2923.

33 Reiss K, Bhakdi S. Pore-forming bacterial toxins and antimicrobial peptides as modulators of ADAM function. Med Microbiol Immunol 2012; 201:419-426.

34 Wilke GA, Bubeck Wardenburg J. Role of a disintegrin and metalloprotease 10 in Staphylococcus aureus $\alpha$-hemolysin-mediated cellular injury. Proc Natl Acad Sci USA 2010; 107:13473-13478.

35 Reiss K, Saftig P. The "a disintegrin and metalloprotease" (ADAM) family of sheddases: physiological and cellular functions. Semin Cell Dev Biol 2009; 20:126-137.

36 Kaczmarek A, Vandenabeele P, Krysko DV. Necroptosis: the release of damage-associated molecular patterns and its physiological relevance. Immunity 2013; 38:209-223.

37 Welz PS, Wullaert A, Vlantis K, et al. FADD prevents RIP3-mediated epithelial cell necrosis and chronic intestinal inflammation. Nature 2011; 477:330-334.

38 Fang DF, He K, Wang N, et al. NEDD4 ubiquitinates TRAF3 to promote CD40-mediated AKT activation. Nat Commun 2014; 5:4513.

(Supplementary information is linked to the online version of the paper on the Cell Research website.) 